\title{
Prognostic variables in patients with primary soft tissue sarcoma of the extremity and trunk treated with neoadjuvant radiotherapy or neoadjuvant sequential chemoradiotherapy
}

\author{
Meena Bedi ${ }^{1}$, David M King ${ }^{2}$, Mikesh Shivakoti ${ }^{3}$, Tao Wang ${ }^{3}$, Eduardo V Zambrano ${ }^{4}$, John Charlson ${ }^{5}$, \\ Donald Hackbarth'², John Neilson ${ }^{2}$, Robert Whitfield ${ }^{6}$ and Dian Wang ${ }^{1 *}$
}

\begin{abstract}
Background: Neoadjuvant radiotherapy (NRT) is an effective strategy to treat soft tissue sarcomas (STS). However, the role of neoadjuvant chemoradiotherapy (NCRT) remains to be determined.

Methods: From May 1999 to July 2010, 112 patients with localized STS of the extremity and trunk who were treated with NRT or NCRT followed by surgery were retrospectively reviewed. Clinical outcomes including overall survival (OS), disease-free survival (DFS), and distant metastasis free survival (DMFS) were calculated using KaplanMeier survival analyses. Prognostic variables were determined by univariate (UVA) and multivariate analyses (MVA).

Results: Median follow-up was 37 months. Median RT dose was 50 Gy. Forty-nine patients received NCRT. Overall limb-preservation rate was $99 \%$ and local control was 97\%. The estimated 3-year OS, DFS, and DMFS were 86\%, $68 \%$, and $72 \%$, respectively. Age was the only variable to predict for OS, DFS and DMFS on UVA. Age $\geq 70$ predicted for poor OS, stage III disease predicted for poor DFS and DMFS, and the addition of chemotherapy predicted for improved DMFS on MVA.
\end{abstract}

Conclusions: Excellent rates of local control and limb-preservation were observed in patients with primary STS treated with neoadjuvant therapy followed by surgery. Neoadjuvant sequential chemotherapy followed by radiotherapy may be considered for young patients with stage III STS.

Keywords: Sarcoma, Preoperative radiation therapy, Preoperative chemotherapy, Prognostic factors, Survival

\section{Introduction}

The management of soft tissue sarcomas (STS) of extremity and trunk has evolved in the past two decades. Limbsalvage surgery combined with either pre-operative or post-operative radiotherapy has now become the standard of care over amputation [1-3]. Although there is no definitive evidence to support a survival benefit of neoadjuvant radiotherapy over adjuvant radiotherapy, results from a recent Phase III study demonstrate a significant advantage of neoadjuvant radiotherapy in the reduction of late radiation morbidities, such as fibrosis, joint stiffness and

\footnotetext{
* Correspondence: dwang@mcw.edu

'Department of Radiation Oncology, Medical College of Wisconsin, 8701

Watertown Plank Rd, Milwaukee, WI 53045, USA

Full list of author information is available at the end of the article
}

edema [4]. Reduction of late radiation morbidities is important in patient's quality of life, as these morbidities are most likely irreversible. Additional advantages of neoadjuvant radiotherapy are a decrease in target volume, radiation dose and tumor seeding during sarcoma resection, as well as an occasional reduction in tumor mass that might facilitate complete resection [5-7].

Neoadjuvant chemoradiotherapy has been utilized in the management of high-risk STS of extremities [8-11]. For instance, neoadjuvant chemotherapy (MAID regimen) interdigitated with neoadjuvant radiotherapy (44 Gy in 22 fractions) has been shown to decrease the rate of distant metastasis (DM) and to increase disease free survival (DFS) and overall survival (OS) in patients with STS of the extremity and trunk with $\geq 8 \mathrm{~cm}$ tumors compared with 
historical control [8]. This prompted RTOG 9514, which was a single-arm phase II trial that enrolled 64 patients with intermediate-to-high grade, $\geq 8 \mathrm{~cm}$ STS of the extremity or torso with expected margin-negative (R0) resection. The 3-year loco-regional failure was $18 \%$ if amputation was considered a failure and $10 \%$ if not. Estimated 5-year rates of DFS, distant metastasis-free survival (DMFS), and OS were $56.1 \%, 64.1 \%$, and $71.2 \%$, respectively. However, significant toxicities associated with this aggressive neoadjuvant chemoradiotherapy regimen have precluded widespread use of this regimen [9].

Neoadjuvant sequential chemoradiotherapy has been known to be less toxic than neoadjuvant concurrent/interdigitated chemoradiotherapy and is commonly used to treat high-risk STS at many institutions. However, it remains to be determined whether this approach provides a survival benefit in patients with STS of the extremity and trunk. In this study, we analyzed a cohort of patients with primary STS of the extremity and trunk who received neoadjuvant radiotherapy with or without sequential neoadjuvant chemotherapy at our institution. The aim of this retrospective study was to determine the clinical and pathologic variables that predict for improved OS, DFS, and DMFS in patients with STS treated with this regimen.

\section{Materials and methods}

This research was reviewed and approved by the Institutional Review Board (IRB) and all investigators completed training in both human research and patient privacy.

\section{Patients}

All patients with primary STS of the upper and lower extremities and trunk who received radiation with or without chemotherapy followed by surgical resection between May 1999 and October 2010 were reviewed. Exclusion criteria included metastatic disease on initial presentation, age $<18$ years old, STS of locations other than the extremity or trunk, recurrent sarcomas at first presentation, and histopathologic types demonstrating rhabdomyosarcoma, extraosseous primitive neuroectodermal tumor, Ewing sarcoma, osteosarcoma, Kaposi's sarcoma, angiosarcoma, aggressive fibromatosis, or dermatofibrosarcoma protuberans. Patients who did not have complete medical records including treatment information and a pathology report, and follow-up of less than 6 months were also excluded. Patients were staged according to the 2009 American Joint Committee on Cancer (AJCC) system seventh edition.

\section{Statistical analysis}

The sample size for this analysis was 112 patients. Potential prognostic variables assessed were location, grade, size, depth, age, percent necrosis on surgical specimens, whether neoadjuvant chemotherapy was administered, and the number of chemotherapy cycles. The 2, 3 and 5-year OS, DFS, and DMFS rates were estimated using the Kaplan-Meier estimate of the survival function. The logrank test was used to compare two survival curves. Univariate and multivariate analyses were performed to determine prognostic variables in correlation with the above survivals. For multivariate analysis, the Cox proportional hazards model was used. All potential risk factors were first tested for affirmation of the potential hazards assumptions. No factors were found to violate the proportional hazards assumption. A step-wise model building procedure was then performed to develop multivariate models for each outcome. For all analyses, the type I error was maintained at 0.05 and all tests were two-sided. All statistical analyses were performed in SAS, ver. 9.1 (Cary, NC).

\section{Results}

\section{Patient characteristics}

There were 112 patients with stage I-III STS of extremity and trunk that were included in this analysis. Median age was 54.5 years old, with a range between 18 to 92 years. Patient characteristics are listed in Table 1.

The most common histology was malignant fibrous histiocytoma (30.3\%). Other histologies included liposarcoma (23.2\%), myxofibrosarcoma (14.3\%), leiomyosarcoma (8.9\%), synovial sarcoma (8.0\%), extraskeletal myxoid chondosarcoma $(2.7 \%)$, malignant peripheral nerve sheath tumor (3.6\%), solitary fibrous tumor (3.6\%), spindle cell sarcoma, NOS (1.8\%), epitheliod sarcoma (1.8\%), and fibrosarcoma (1.8\%). Nineteen patients (17\%) had low grade, $6(5 \%)$ had intermediate grade, and 87 (78\%) had high grade disease.

All patients underwent external beam radiotherapy followed by limb-sparing, wide-local excision. The median

Table 1 Patient characteristics

\begin{tabular}{lcc}
\hline \multirow{2}{*}{ Tumor Stage } & \multicolumn{1}{c}{ Frequency (\%) } \\
\cline { 2 - 3 } & \multicolumn{1}{|l}{ III } & 17 \\
\cline { 2 - 3 } Histology & Undifferentiated/MFH & 14 \\
\cline { 2 - 3 } & Liposarcoma & 69 \\
\cline { 2 - 3 } & Myxofibrosarcoma & 35 \\
\cline { 2 - 3 } & Leiomyosarcoma & 26 \\
\cline { 2 - 3 } & Synovial Cell Sarcoma & 17 \\
\cline { 2 - 3 } & Other & 10 \\
\hline Location & Trunk & 10 \\
\cline { 2 - 3 } & Extremity & 20 \\
\hline Grade & Low & 17 \\
\cline { 2 - 3 } & Intermediate & 78 \\
\cline { 2 - 3 } & High & 17 \\
\hline
\end{tabular}


dose administered was 50 Gy in 25 fractions using either three-dimensional radiotherapy or intensity modulated radiation treatment. Of the patients with accessible radiation records, a three-dimensional technique was used in $79.4 \%$ and IMRT was used in $20.6 \%$ of patients. Surgical resection was performed 4-8 weeks after completion of radiation treatment. Negative margins, which are defined as tumor on ink, were achieved in $92 \%$ of all cases upon wide local excision. The limb-preservation rate was $99 \%$. One patient underwent an amputation for a failed infected reconstruction with vascular flap. Immediate flap reconstructions were performed in 53 (47.5\%) of patients.

Forty-nine patients (40\%) received neoadjuvant chemotherapy prior to the initiation of radiation. All chemotherapy was doxorubicin and ifosfamide-based regimens (Table 2). Twenty-eight patients $(57 \%)$ received 3 or more cycles of chemotherapy, 20 of these patients received less than 3 cycles of chemotherapy, and 63 (56\%) patients received no chemotherapy. The number of cycles of chemotherapy was unknown in one patient. Neoadjuvant chemotherapy was often given to young patients $(<70$ years old) with clinical stage III (large, deep, intermediateto-high grade) STS. Neoadjuvant chemotherapy was given to 4 out of $48(8.3 \%)$ patients $\geq 70$ years old compared to 27 out of 64 patients $(42.2 \%)$ who were $<70$ years old and did not receive chemotherapy $(\mathrm{p}<0.0001)$.

Adjuvant chemotherapy was also delivered in 14 patients who received neoadjuvant chemotherapy. Twelve patients received a doxorubicin-ifosfamide based regimen, one patient received an epirubicin-ifosfamide based regimen, and one patient received adjuvant chemotherapy at an outside clinic, but the regimen was not available for review. One patient received five cycles of adjuvant chemotherapy, 5 patients received two cycles, 2 patients received three cycles, and the remaining 6 received an undetermined number of cycles.

Table 2 Demographics of patients receiving neoadjuvant chemotherapy

\begin{tabular}{cccc}
\hline Variable & & NRT & NCRT \\
\hline Age & $\leq 70$ & $39(45 \%)$ & $48(55 \%)$ \\
\cline { 2 - 4 } & $>70$ & $21(84 \%)$ & $4(16 \%)$ \\
\hline Stage & 1 & $18(9.5 \%)$ & $1(5 \%)$ \\
\cline { 2 - 4 } & $\| 11$ & $10(62.5 \%)$ & $6(37.5 \%)$ \\
\cline { 2 - 4 } & Low & $33(43 \%)$ & $44(57 \%)$ \\
\cline { 2 - 4 } & Intermediate & $4(67 \%)$ & $1(5 \%)$ \\
\cline { 2 - 4 } & High & $38(44 \%)$ & $49(33 \%)$ \\
\hline Size & $\leq 5 \mathrm{~cm}$ & $3(30 \%)$ & $7(70 \%)$ \\
\cline { 2 - 4 } & $>5 \mathrm{~cm}$ & $49(48 \%)$ & $53(52 \%)$ \\
\hline
\end{tabular}

NRT: neoadjuvant radiotherapy. NCRT: neoadjuvant chemoradiotherapy.

\section{Pathologic outcomes}

Percent necrosis was documented in 102 of the 112 patients in our database. The original pathology reports were centrally reviewed by our sarcoma pathologist (EZ), who was blind to clinical outcomes. Ten patients had $100 \%$ necrosis of their tumor after neoadjuvant treatment, 14 patients had $\geq 99 \%$ necrosis, 23 patients had $\geq 95 \%$ necrosis, 20 patients had $80-94 \%$ necrosis, 20 patients had 50-79\% necrosis, and 39 patients had less than $50 \%$ necrosis of their tumor.

\section{Clinical outcomes}

Median follow-up was 3.1 years. Eight patients were lost to follow-up. The local control rate for the entire study population was $97 \%$ at this follow-up. Two of the 3 patients that failed locally did so after the development of distant metastasis. The distant metastasis rate for the study group was $27.6 \%$. The median time to death was 23.5 months. The median time to disease progression was 16.3 months. The median time to distant metastasis was 15.6 months.

The median OS, DFS and DMFS was > 124 months (Figure 1A, 1B, and 1C), with a 3-year OS of $85.5 \%$, 3year DFS of $68.3 \%$, and 3-year DMFS of $71.7 \%$.

There was no significant difference in the OS $(\mathrm{p}=0.42)$, DFS $(\mathrm{p}=0.29)$, and DMFS $(\mathrm{p}=0.12)$ between T1 (tumor $\leq 5 \mathrm{~cm}$ ) and T2 (tumor $>5 \mathrm{~cm}$ ) STS. There was no significant difference in OS $(\mathrm{p}=0.73)$, DFS $(\mathrm{p}=0.41)$, and DMFS $(\mathrm{p}=0.48)$ between deep and superficial sarcomas. There 3-year OS for low, intermediate and high grade disease was $93.7 \%, 83.3 \%$ and $84.6 \%$, respectively $(\mathrm{p}=0.5)$. The 3-year DFS for low, intermediate and high grade disease was $83.1 \%, 76.2 \%$, and $64.9 \%$, respectively $(\mathrm{p}=0.2)$. The 3 -year DMFS was $82.6 \%, 71 \%$ and $69 \%$, respectively $(\mathrm{p}=0.2)$.

Necrosis assessed on pathology from the surgical specimen was grouped according to the degree of necrosis after the central pathology review. There was no significant difference in OS $(\mathrm{p}=0.4)$, DFS $(\mathrm{p}=0.37)$, and DMFS $(\mathrm{p}=0.71)$ in patients who had $\geq 95 \%$ vs. $\geq 80 \%-94 \%$ vs. $\geq 50 \%-79 \%$ vs. $<50 \%$ necrosis.

On univariate analysis, age $<70$ predicted for OS $(\mathrm{p}=0.019)$, DFS $(\mathrm{p}=0.037)$, and DMFS $(\mathrm{p}=0.021)$. Stage also predicted for DMFS on UVA $(p=0.017)$. No other variable was significant for OS, DFS or DMFS. On multivariate analysis, age $\geq 70$ was significant for poor OS $(\mathrm{p}=0.026)$, stage III disease was significant for poor DFS $(\mathrm{p}=0.041)$ and DMFS $(\mathrm{p}=0.004)$, and the addition of neoadjuvant chemotherapy prior to preoperative radiotherapy was significant for improved DMFS ( $\mathrm{p}=0.041)$ (Figure 2).

\section{Discussion}

High local control rates have been demonstrated using multimodality therapy for STS. Yang et al., showed that the 

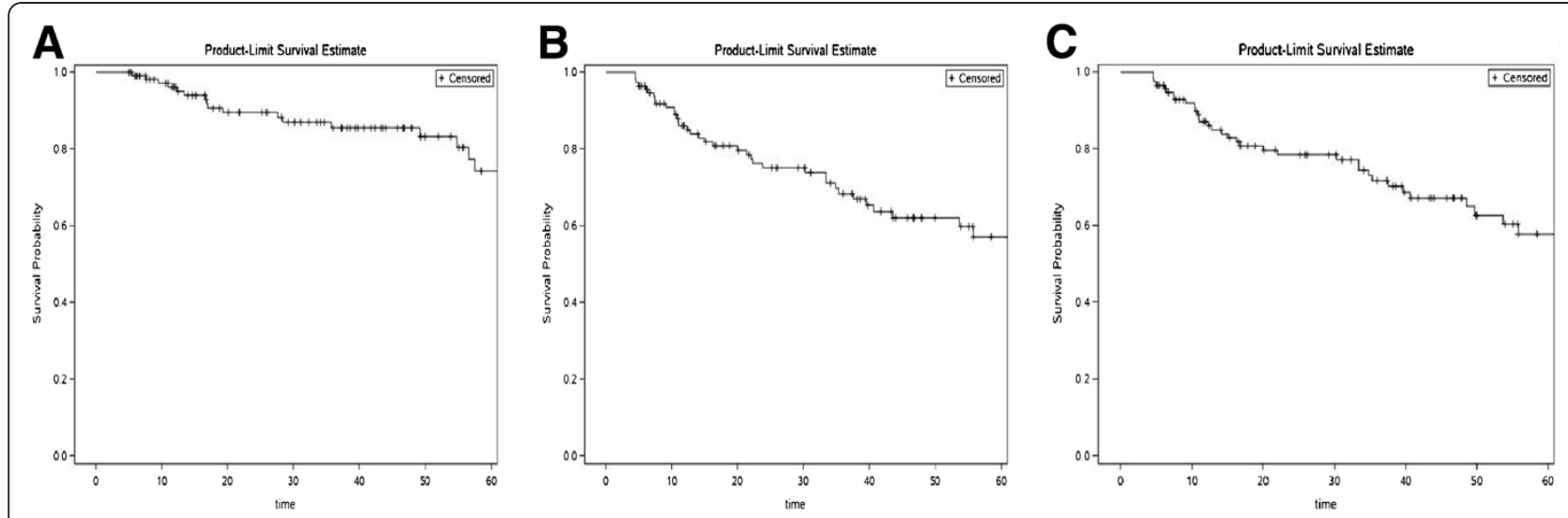

Figure 1 Overall survival (A), disease-free survival (B), distant metastasis free survival (C) of the entire group.

addition of post-operative radiation to chemotherapy improved local control rates in both high and low grade tumors [12]. Several other studies have demonstrated high local control rates of approximately $90 \%$ at 5 years with preoperative radiotherapy with or without chemotherapy [4,13-16] (Table 3). Brant, et al., demonstrated a 91\% local control rate in 58 patients with STS of the extremity or trunk who were treated with preoperative radiation alone followed by surgical resection. The University of Florida series was recently updated with an overall local control rate of $94 \%$. Similarly, Barkley, et al. also had high rates of local control $[13,14,17]$. Studies that have compared preoperative radiation to post-operative radiation have also shown equivalent outcomes for local control $[4,15,16]$. Similarly, this study demonstrates a high local control rate of $97 \%$ at a median follow-up of 3.1 years for those treated with preoperative radiation therapy followed by limb-sparing surgery. This suggests that neoadjuvant radiotherapy combined with surgical resection does not compromise local control in STS of the extremity and trunk.

Neoadjuvant chemotherapy is often preferred over adjuvant chemotherapy, although there is no level one evidence to support a survival advantage with the use of neoadjuvant chemotherapy in patients with STS. The Sarcoma Meta-analysis of STS patients enrolled into 14 randomized trials revealed that adjuvant chemotherapy was found to improve local recurrence-free survival by $6 \%$, distant and overall recurrence-free survival by $10 \%$ [18]. However, proponents for neoadjuvant chemotherapy argue that oncologists are able to monitor response and alter or terminate therapy in patients who do not appear to be deriving any benefit. Gortzac, et. al reported results of a phase II study that examined patients with high-risk sarcomas who were randomized to surgery alone vs. three cycles of doxorubicin and ifosfamide prior to surgery. After a median follow-up of 7.3 years, the addition of

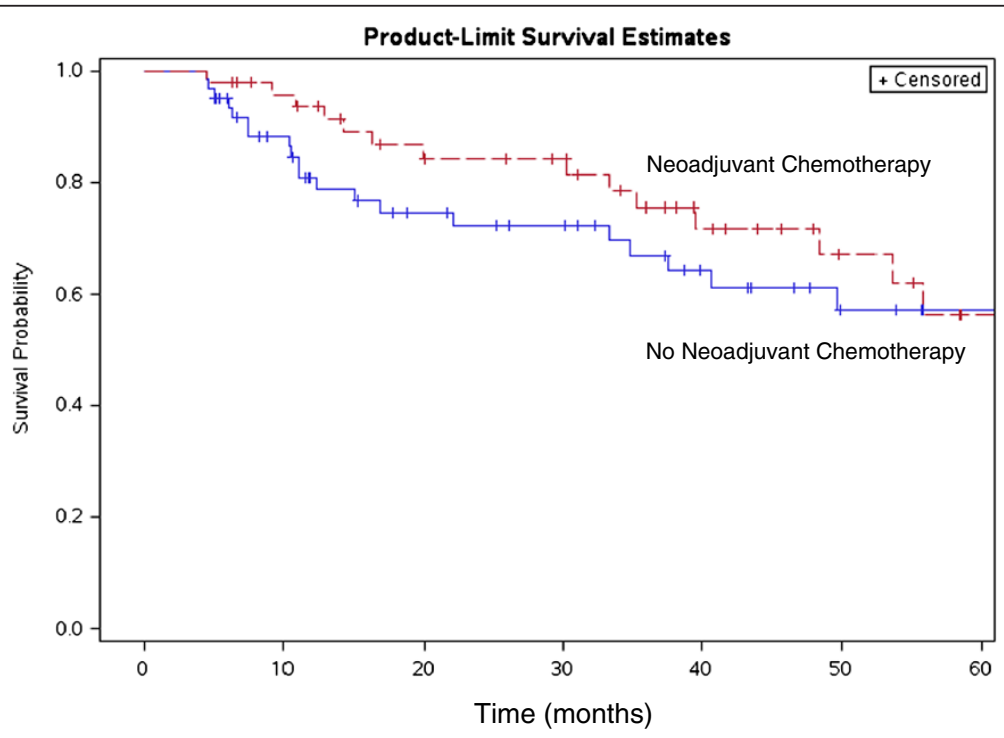

Figure 2 Distant metastasis free survival (DMFS) of patients with neoadjuvant chemotherapy versus without neoadjuvant chemotherapy. 
Table 3 Local control with neoadjuvant radiotherapy for primary soft tissue sarcomas of extremity and/or trunk

\begin{tabular}{|c|c|c|c|c|c|}
\hline Study & Institution & Number of pre-op patients \& follow-up & Chemotherapy included & Median RT dose (Gy) & Local control \\
\hline \multirow[t]{2}{*}{ Dagan, et al. } & $U$ of Florida & 317 & Yes & 50.4 & $94 \%$ \\
\hline & & $4.7 y$ & & & \\
\hline \multirow[t]{2}{*}{ O'Sullivan, et al. } & $\mathrm{NCl}$ & 93 & No & 50 & $93 \%$ \\
\hline & & $3.3 \mathrm{y}$ & & & \\
\hline \multirow[t]{2}{*}{ Sampath, et al. } & Utah & 293 & Yes & 50.4 & $93 \%$ \\
\hline & & $5.25 \mathrm{y}$ & & & \\
\hline \multirow[t]{2}{*}{ Zagars, et al. } & MDACC & 271 & Yes & 50 & $87 \%$ \\
\hline & & $6 y$ & & & \\
\hline \multirow[t]{2}{*}{ Bedi, et al. } & MCW & 112 & Yes & 50 & $97 \%$ \\
\hline & & $3.1 \mathrm{y}$ & & & \\
\hline
\end{tabular}

chemotherapy failed to show any benefit in DFS or OS [19]. Authors concluded that this study did not have enough power to address survival. Therefore, neoadjuvant chemotherapy alone should not be routinely recommended to all patients with STS.

The results from this study show that the use of neoadjuvant sequential chemotherapy was associated with a higher DMFS in patients $<70$ years old with clinical stage III STS of the extremity and trunk. This is similar to the results of other studies compared with historical controls $[8,9]$. The mechanism of biological synergy from neoadjuvant sequential chemoradiotherapy remains unknown. Considering the rarity of STS and uncertain response of some STS to neoadjuvant chemotherapy, the authors would therefore recommend neoadjuvant sequential chemoradiotherapy be considered in treating patients $<70$ years old with stage III STS in centers with considerable multidisciplinary expertise and experience in managing these patients and where such an approach can be discussed for appropriate patients in the setting of a multidisciplinary sarcoma tumor board. As such, close monitoring of these patients are essential during the course of neoadjuvant chemotherapy. At our institution, patients $<70$ years old with stage III STS of the extremity and trunk are jointly evaluated by all disciplines.

The decision to administer neoadjuvant chemotherapy prior to radiation is dependent on several factors. Grade, tumor size, and histology all are taken into account, as well as patient factors such as performance status, cardiovascular and renal functioning and patient preference. Typically, chemotherapy is recommended in high grade STS with certain histologies that are $\geq 5 \mathrm{~cm}$ in size or deep tumors. These histologies include synovial sarcoma, myxoid liposarcoma, dedifferentiated liposarcoma, poorly differentiated STS, and leiomyosarcoma. These patients are also offered neoadjuvant sequential adriamycinifosfamide chemotherapy for three cycles. These patients might receive immediate preoperative radiotherapy due to significant tumor pain or growth after 1 or 2 cycles of chemotherapy. All patients will have a CT scan of the chest and an MRI of the primary sarcoma after 2 cycles of chemotherapy. Those patients whose tumors are stable or shrink would proceed with the third cycle of neoadjuvant chemotherapy prior to preoperative radiotherapy. All of the above scenarios should be extensively discussed with these patients prior to initiating treatment.

Prognostic variables have been investigated in many retrospective studies [20-29]. Stevanovski, et al. have demonstrated that stage is prognostic in OS; other studies show that grade, size, and depth of tumor all are prognostic in terms of OS, disease-specific survival, and DMFS [19-25]. In this study, the UVA showed age $\geq 70$ was the only variable that predicted for OS, DFS, and DMFS, and stage III disease predicted for poor DMFS. On MVA, age $\geq 70$ was significant for poor OS, stage III disease was significant for poor DFS and DMFS, and the addition of neoadjuvant chemotherapy prior to preoperative radiotherapy was significant for improved DMFS.

Age at diagnosis has been demonstrated to be a prognostic indicator in several other studies [21,27-29]. Both Pisters, et $a l$. and Gronchi et $a l$., showed decreased local control rates in patients with STS [21,27]. A study done by Collin et $a l$., revealed that survival was also impacted in patients who were $>53$ years old at diagnosis, and Kaytan, et al. also showed poorer survival in patients $>50$ years of age $[28,29]$. These studies corroborate our finding that advanced age at diagnosis may portend to poorer outcomes. Furthermore, a 12-year sarcoma-specific death nomogram has been created by Kattan and colleagues, which uses age as one of the nomogram predictor variables [30].

Treatment-induced tumor necrosis due to radiation with or without chemotherapy has been sparsely reported. Eliber, et al., showed improved 5-year survival rates of $80 \%$ vs. $62 \%$ in tumors with near-complete necrosis [11]. Similarly, MacDermed, et al. demonstrated a 5-year freedom-from -distant metastasis rate of $84.6 \%$ in patients who had a high rate of necrosis after treatment $(\geq 90 \%)$ vs $19.9 \%$ in tumors that had less extensive necrosis [31]. However, a 
prospective phase II RTOG study on STS demonstrated that a near complete necrosis after neoadjuvant interdigitated chemoradiotherapy did not predict for any survival outcomes [8]. Similarly, results of this study showed the percent of necrosis after preoperative radiotherapy with or without chemotherapy did not impact OS, DFS, or DMFS. The reasons for the differences from our study are unclear and could be secondary to tissue sample selection that is not able to be examined in a retrospective study. There are also several issues about documenting percent of necrosis after neoadjuvant treatment for STS. First, there are no methods to differentiate between treatment-induced necrosis and tumor-related necrosis either through imaging studies or pathology examination. Second, the preand post-treatment percent of necrosis were not able to be compared in our study and other studies [11,31]. Third, percent of necrosis was not uniformly evaluated in above studies due to the large size of STS, as there was a technical challenge to evaluate entire specimen of large STS. Finally, different from many other types of human malignancy, high grade and large size are often associated with a significant increase in STS necrosis at initial diagnosis. This observation is confirmed in our study.

Limitations of this study include the inherent biases of a retrospective review and the relatively small sample size. Moreover, there were a heterogeneous group of tumors analyzed.

\section{Conclusions}

Excellent rates of local control and limb-preservation were observed in patients with primary STS of the extremity and trunk treated with neoadjuvant radiation or neoadjuvant sequential chemoradiation followed by surgery. Age $\geq 70$ years old was the only variable alone found to be prognostic for poor OS, DFS, and DMFS and stage III disease predicted for poor DMFS on UVA. Results of the MVA suggest that neoadjuvant sequential chemotherapy followed by radiation may be considered for young patients ( $<70$ years old) with stage III STS of the extremity and trunk.

\section{Competing interest}

The authors' declare that they have no competing interest.

\section{Authors' contributions}

MB, DMK and DW were responsible for data collection, study concept and design, creating figures and tables, as well as manuscript preparation and revision. MS and TW were responsible for statistical analysis and figure creation with help from the statistical software. DMK, JC, DAH, JN, RW and DW staged each patient's disease, performed surgical resections for pathology to review, treated patient's according to recommendations and revised the manuscript. EAZ was responsible for pathological re-review of each case in this study and revisions of the manuscript. DW had the origina data and was responsible supervision and confirming the final manuscript. All authors have read and approved the final manuscript.

\section{Author details}

'Department of Radiation Oncology, Medical College of Wisconsin, 8701 Watertown Plank Rd, Milwaukee, WI 53045, USA. ${ }^{2}$ Department of Orthopaedic Surgery, Medical College of Wisconsin, Milwaukee, WI, USA.

${ }^{3}$ Department of Biostatistics Center, Medical College of Wisconsin, Milwaukee, WI, USA. ${ }^{4}$ Department of Pathology, Medical College of Wisconsin, Milwaukee, WI, USA. ${ }^{5}$ Department of Medical Oncology, Medical College of Wisconsin, Milwaukee, WI, USA. 'Department of Plastic Surgery, Medical College of Wisconsin, Milwaukee, WI, USA.

Received: 3 October 2012 Accepted: 5 March 2013

Published: 14 March 2013

\section{References}

1. Bowden $L$, Booher RJ: The principles and technique of resection of soft parts for sarcoma. Surgery 1958, 44:963-977.

2. Simon MA, Enneking WF: The management of soft-tissue sarcomas of the extremities. J Bone Joint Surg Am 1976, 58:317-327.

3. Rosenberg SA, Tepper J, Glatstein $\mathrm{E}$, et al: The treatment of soft-tissue sarcomas of the extremities: prospective randomized evaluations of (1) limb-sparing surgery plus radiation therapy compared with amputation and (2) the role of adjuvant chemotherapy. Ann Surg 1982, 196:305-315.

4. O'Sullivan B, Davis AM, Turcotte R, et al: Preoperative versus postoperative radiotherapy in soft-tissue sarcoma of the limbs: a randomised trial. Lancet 2002, 359:2235-2241.

5. Nielsen OS, Cummings B, O'Sullivan B, et al: Preoperative and postoperative irradiation of soft tissue sarcomas: effect of radiation field size. Int J Radiat Oncol Biol Phys 1991, 21:1595-1599.

6. Sadoski C, Suit HD, Rosenberg A, et al: Preoperative radiation, surgical margins, and local control of extremity sarcomas of soft tissues. J Surg Oncol 1993, 52:223-230.

7. Wilson AN, Davis A, Bell RS, et al: Local control of soft tissue sarcoma of the extremity: the experience of a multidisciplinary sarcoma group with definitive surgery and radiotherapy. Eur J Cancer 1994, 30A:746-751.

8. DeLaney TF, Spiro IJ, Suit HD, et al: Neoadjuvant chemotherapy and radiotherapy for large extremity soft-tissue sarcomas. Int J Radiat Oncol Biol Phys 2003, 56:1117-1127.

9. Kraybill WG, Harris J, Spiro IJ, et al: Phase II study of neoadjuvant chemotherapy and radiation therapy in the management of high-risk, high-grade, soft tissue sarcomas of the extremities and body wall: Radiation Therapy Oncology Group Trial 9514. J Clin Oncol 2006, 24:619-625.

10. Eilber FR, Morton DL, Eckardt J, et al: Limb salvage for skeletal and soft tissue sarcomas. Multidisciplinary preoperative therapy. Cancer 1984, 53:2579-2584

11. Eilber FC, Rosen G, Eckardt J, et al: Treatment-induced pathologic necrosis: a predictor of local recurrence and survival in patients receiving neoadjuvant therapy for high-grade extremity soft tissue sarcomas. J Clin Oncol 2001, 19:3203-3209.

12. Yang JC, Cheng AE, Baker AR, et al: Randomized prospective study of the benefit of adjuvant radiation therapy in the treatment of soft tissue sarcomas of the extremity. J Clin Oncol 1998, 16:197-203.

13. Brant TA, Parsons JT, Marcus RB Jr, et al: Preoperative irradiation for soft tissue sarcomas of the trunk and extremities in adults. Int J Radiat Oncol Biol Phys 1990, 19:899-906.

14. Barkley HT Jr, Martin RG, Romsdahl MM, et al: Treatment of soft tissue sarcomas by preoperative irradiation and conservative surgical resection. Int J Radiat Oncol Biol Phys 1988, 14:693-699.

15. Sampath $S$, Schultheiss TE, Hitchcock YJ, et al: Preoperative versus postoperative radiotherapy in soft-tissue sarcoma: multi-institutional analysis of 821 patients. Int J Radiat Oncol Biol Phys 2011, 81:498-505.

16. Zagars GK, Ballo MT, Pisters PW, et al: Preoperative vs. postoperative radiation therapy for soft tissue sarcoma: a retrospective comparative evaluation of disease outcome. Int J Radiat Oncol Biol Phys 2003, 56:482-488

17. Dagan $R$, Indelicato $D J, M c G e e ~ L$, et al: The significance of marginal excision after preoperative radiation therapy for soft tissue sarcoma of the extremity. Cancer 2012, 118:3199-3207.

18. Sarcoma Meta-analysis Collaboration (SMAC): Adjuvant chemotherapy for localised soft tissue sarcoma in adults (Cochrane Review). Oxford, United Kingdom: The Cochrane Library, Update Software; 2003. 
19. Gortzak E, Azzarelli A, Buesa J, et al: A randomised phase II study on neoadjuvant chemotherapy for 'high-risk' adult soft-tissue sarcoma. Eur J Cancer 2001, 37:1096-1103.

20. Stefanovski PD, Bidoli E, De Paoli A, et al: Prognostic factors in soft tissue sarcomas: a study of 395 patients. Eur J Surg Oncol 2002, 28:153-164.

21. Pisters PW, Leung DH, Woodruff J, et al: Analysis of prognostic factors in 1,041 patients with localized soft tissue sarcomas of the extremities. J Clin Oncol 1996, 14:1679-1689.

22. Zagars GK, Ballo MT, Pisters PW, et al: Prognostic factors for diseasespecific survival after first relapse of soft-tissue sarcoma: analysis of 402 patients with disease relapse after initial conservative surgery and radiotherapy. Int I Radiat Oncol Biol Phys 2003, 57:739-747.

23. Coindre JM, Terrier P, Bui NB, et al: Prognostic factors in adult patients with locally controlled soft tissue sarcoma. A study of 546 patients from the French Federation of Cancer Centers Sarcoma Group. J Clin Oncol $1996,14: 869-877$.

24. Singer $\mathrm{S}$, Corson JM, Gonin R, et al: Prognostic factors predictive of survival and local recurrence for extremity soft tissue sarcoma. Ann Surg 1994, 219:165-173.

25. Stojadinovic A, Leung DH, Allen P, et al: Primary adult soft tissue sarcoma: time-dependent influence of prognostic variables. J Clin Oncol 2002, 20:4344-4352.

26. Kolovich GG, Wooldridge AN, Christy JM, et al: A retrospective statistical analysis of high-grade soft tissue sarcomas. Med Oncol 2012, 29:1335-1344

27. Gronchi A, Miceli R, Colombo C, et al: Primary extremity soft tissue sarcomas: outcome improvement over time at a single institution. Ann Oncol 2011, 22:1675-1681.

28. Collin C, Gadbold J, Hajdu S, et al: Localized extremity soft tissue sarcoma. An analysis of factors affecting survival. J Clin Oncol 1987, 5:601-612.

29. Kaytan E, Yaman F, Cosar R, et al: Prognostic factors in soft tissue sarcomas. Am J Clin Oncol 2003, 26:411-415.

30. Kattan M, Leung D, Brennan M: Post-operative Nomogram for 12-year sarcoma specific survival. J Clin Oncol 2002, 20:791-796.

31. MacDermed DM, Miller LL, Peabody TD, et al: Primary tumor necrosis predicts distant control in locally advanced soft-tissue sarcomas after preoperative concurrent chemoradiotherapy. Int I Radiat Oncol Biol Phys 2010, 76:1147-1153.

doi:10.1186/1748-717X-8-60

Cite this article as: Bedi et al:: Prognostic variables in patients with primary soft tissue sarcoma of the extremity and trunk treated with neoadjuvant radiotherapy or neoadjuvant sequential chemoradiotherapy. Radiation Oncology 2013 8:60.

\section{Submit your next manuscript to BioMed Central and take full advantage of:}

- Convenient online submission

- Thorough peer review

- No space constraints or color figure charges

- Immediate publication on acceptance

- Inclusion in PubMed, CAS, Scopus and Google Scholar

- Research which is freely available for redistribution 